\title{
FIRST WHOLLY-ANALYTICAL GAS VOLUME FRACTION MODEL FOR VIRTUAL MULTIPHASE FLOW METERING PETROLEUM INDUSTRY APPLICATIONS
}

\author{
ANAND S. NAGOO \\ Nagoo \& Associates LLC, Austin, Texas, USA.
}

\begin{abstract}
In this seminal contribution, the world's first wholly-analytical gas volume fraction multiphase flow model is formulated and demonstrated in virtual flow meter and production allocation field applications for its differentiated ability to achieve improved reliability of phase flow rate calculations given pressure and temperature measurements at two different locations along multiphase production systems. The presented simple gas volume fraction equation is explicit in form and is validated against both lab data and oilfield flowline data. A crucial requirement for differential pressure flow meters for multiphase production systems, particularly wet gas systems in annular and annular-mist flows, is the calculation of the averaged gas volume fraction. Additional calculations include multidirectional entrainment calculations, which strongly affect the simultaneous entrainment of liquids in the gas phase and the gas in the liquid phases. Historically, prior published gas volume fraction two-phase flow models had closure relations and artificial adjustment (fitting) factors linked to controlled lab-scale conditions involving immiscible fluids that bear no resemblance to the complex petroleum mixtures undergoing phase change in uncontrolled long wellbore and flowline environments. Thus, ambiguous extrapolations were necessary leading to increased uncertainties. Using an asymptotic approximation analysis approach, an analytical gas volume fraction equation is derived that overcomes this empirical-based restriction. In terms of comprehensive validation, the presented analytical gas volume fraction equation is demonstrated first for its ability to reliably reproduce over 2600 two-phase annular and annular-mist flow experimental datasets inclusive of circular and non-circular conduits. Secondly, readily available published experimental data of both constant-diameter as well as variable-diameter sub-critical to critical choke two-phase flows are used for model validation in scenarios involving different flow obstructions. Lastly, an offshore subsea flowline dataset is used to demonstrate the improved reliability of the new equation at field-scale operational conditions.

Keywords: Multiphase Flow Metering, Oil Production Allocation, Offshore Flowline and Onshore Production Systems, Volume Fractions and Flow Rates Prediction, Wet Gas Virtual Flow Metering.
\end{abstract}

\section{INTRODUCTION}

The well-recognized needs for consistently accurate multiphase flow metering (MPFM) in the petroleum industry stem from the fact that a reliable determination of the in-line flow rates of the unprocessed oil, gas and water phases, is necessary for allocating the production from oil and gas assets, for well testing and for continuous monitoring and optimization of production. In contrast to partially and fully separated multiphase flow meters such as test separators on petroleum production systems, in-line MPFM measurements and calculations are meant to determine the oil, water and gas flow rates without any processing or conditioning. Such in-line MPFM technologies and applications have been in wide-spread use since the early 1990's [1]. A good review and description of various available in-line MPFM technologies and devices can be found in [2]. Furthermore, detailed descriptions of prior theories and algorithms used in either the inferred measurements of physical flow meters or in physics-based flow metering models (i.e. virtual flow meters, VFM), can be found in [3] and [2]. In a more recent application of MPFM, an example of using physics-based flow metering models in combination with automatic calibration using artificial data-fitting factors derived from Data Analytics (i.e. 'smart' virtual flow meters, SVFM), can be found in [4]. 
Among the various approaches used to measure or calculate the three-phase flow rates of a production system, it is well-recognized that those approaches involving the calculation of gas volume fraction will lead to a more consistently reliable prediction of phase flow rates due to the inherent relationship between phase volume fractions, velocities and pressure gradient [2]. This is no surprise since this relationship is merely a manifestation of the law of conservation of total momentum of the flowing multiphase mixture. In particular, MPFM calculation algorithms utilize different forms of this relationship in combination with a viscous loss coefficient and the discernible pressure drop across restricted multiphase flows (such as through nozzles, orifices and critical/sub-critical chokes) to obtain more reasonable estimates of flow rates. The question then arises as to which gas volume fraction model to use in a MPFM calculation algorithm for the best reliably predictive results? The answer to this question is the scope of this work.

We emphasize here that our aim is to avoid empirical lab-based correlations for gas volume fraction (i.e. avoid the numerous scaling problems from lab to field) and to avoid calibration in any form since all calibration involving data-fitting decreases predictability since they increase unknowns (increased fitting factors) and lock in the predictive path of models. The practical benefit of a reliably accurate calculation of gas volume fraction for use in VFM or MPFM applications will be to enhance the predictive capability of such devices/algorithms. Additionally, reliable multiphase flow metering calculations present a cost-effective solution for monitoring production at any point along the production system, either standalone or as back-up to installed multiphase flow metering equipment, even in environments where pressure and temperature sensors in wells or flowlines can fail over time.

Last, although the emphasis of this work is on the gas volume fraction analytical model development, other important multiphase calculations that must necessarily go into VFM or MPFM algorithm routines, such as multidirectional entrainment and the thermodynamic conversion of volumetric phase flow rates at standard conditions to in-situ mass flow rates can be found in [5] and [6], respectively. For the generation of all simulation results in this paper, we utilize the analytical multiphase flow methods found in [6].

\section{DERIVATION OF THE ANALYTICAL GAS VOLUME FRACTION MODEL}

Considering above, a correlation-free and mathematically consistent model for gas volume fraction (or gas void fraction) will significantly improve the predictive accuracy and stability of multiphase flow meter calculation algorithms. Firstly, such a model will avoid the large, ambiguous inaccuracies that arise from scaling lab-based low pressure and temperature experiments with immiscible (irrelevant) fluids to field scale environments at high pressure and temperature with complex miscible (petroleum) fluids. Secondly, such a model will avoid artificial data-fitting factors of automated or manual calibration routines, regardless of whether the fitting factors come from tuning/training datasets or not. Indeed, more calibration increases unknowns (more fitting factors) and decreases model predictability in scenarios outside of tuning datasets. Thirdly, such a model must be smooth, continuous and differentiable to enable its use in inverse differential pressure flow meter algorithms that utilize pressure drops to arrive at unique solutions to the in-situ mass flow rates. To this end, an asymptotic approximation analysis is invoked below to derive such an analytical gas volume fraction model.

In continuation of the specific terminology and pipe fractional flow language of [7], for a generic heavier phase 1 (e.g. a liquid) and a generic lighter phase 2 (e.g. a gas), similar to the slip ratio between phase 2 and phase $1, \mathrm{H}_{2,1}$, we can define another dimensionless velocity ratio which we call the relative velocity slip ratio, $\Omega_{2,1}$, which is the ratio of the relative velocity to the mixture velocity of the flowing two-phase mixture: 


$$
\Omega_{2,1}=\frac{\overbrace{\left\langle\left\langle v_{2}\right\rangle\right\rangle-\left\langle\left\langle v_{1}\right\rangle\right\rangle}^{\text {relative velocity }}}{\left\langle u_{\text {mix }}\right\rangle}
$$

In eqn. (1), $\left\langle\left\langle v_{2}\right\rangle\right\rangle$ is the averaged in-situ velocity of phase $2,\left\langle\left\langle v_{1}\right\rangle\right\rangle$ is the averaged in-situ velocity of phase 1 , and $\left\langle u_{\text {mix }}\right\rangle$ is the mixture velocity $=\left\langle\left\langle v_{2}\right\rangle\right\rangle\left\langle s_{2}\right\rangle+\left\langle\left\langle v_{1}\right\rangle\right\rangle\left(1-\left\langle s_{2}\right\rangle\right)$ where $\left\langle s_{2}\right\rangle$ is the averaged phase 2 volume fraction or averaged gas volume fraction in a gas-liquid flow. Therefore, we see with this simple definition, all the in-situ velocities and volume fractions are captured. This is not the case with the slip ratio definition, $\mathrm{H}_{2,1}$, which only captures the slip of one phase relative to the other. As noted in [7], from a transport phenomena perspective, the objectively measurable changes in the in-situ phase velocities and volume fractions associated with each flow pattern are the fundamental physical quantities that govern the transport processes of the multiphase flow. It is these transport processes that drive the different mass, momentum and energy exchanges occurring during flow. This is why flow patterns matter because they represent the visual (spatio-temporal) manifestations of the measurable changes in the in-situ velocities and volume fractions during the multiphase flow.

Now, let's start our derivation by postulating what the upper limit (the upper asymptote) of eqn. (1) would look like. In this limit, in terms of the physical mechanisms at play, phase 1 (the liquid phase in the case of gas-liquid flow) is entraining fully into phase 2 (the gas) and therefore, regardless of the other velocities in the multiphase flow, $\left\langle\left\langle v_{1}\right\rangle\right\rangle$ will tend to $0,\left\langle s_{2}\right\rangle$ will tend to 1 and thus $\left\langle\left\langle v_{2}\right\rangle\right\rangle$ will tend to $\left\langle u_{2}\right\rangle$, the superficial velocity of phase 2 . This can be expressed as:

$$
\Omega_{2,1}=\frac{\left\langle\left\langle v_{2}\right\rangle\right\rangle-\left\langle\left\langle v_{1}\right\rangle\right\rangle}{\left\langle u_{\text {mix }}\right\rangle} \stackrel{\begin{array}{c}
\text { in an upper } \\
\text { flow limit }
\end{array}}{\rightarrow} \frac{\overbrace{\left\langle\left\langle v_{2}\right\rangle\right\rangle}^{\begin{array}{c}
\text { approches } \\
\text { in upper limit }
\end{array}}-\overbrace{\left\langle\left\langle u_{1}\right\rangle\right\rangle}^{\begin{array}{c}
\text { relatively negligible } \\
\text { in upper limit }
\end{array}}}{\left\langle u_{\text {mix }}\right\rangle}=f_{2}
$$

In eqn. (2) above, $f_{2}$ is the flowing fraction of phase $2=\left\langle u_{2}\right\rangle /\left\langle u_{\text {mix }}\right\rangle$. Now, if we re-express eqn. (1) in the dimensionless terms of $f_{2}$ and $\left\langle s_{2}\right\rangle$, we get:

$$
f_{2}=\left(1+\left(1-\left\langle s_{2}\right\rangle\right) \Omega_{2,1}\right)\left\langle s_{2}\right\rangle
$$

It is noteworthy at this juncture to point out that eqn. (3) above is the same as eqn. (5) of [7] but this time expressed in terms of the dimensionless relative velocity ratio form. Substituting eqn. (2) in eqn. (3), we will then arrive at:

$$
f_{2}=\frac{\left\langle s_{2}\right\rangle}{1-\left\langle s_{2}\right\rangle\left(1-\left\langle s_{2}\right\rangle\right)}
$$

And equivalently, solving for $\left\langle s_{2}\right\rangle$ (i.e. the gas volume fraction in a gas-liquid flow) in eqn. (4), we arrive at the analytical expression for $\left\langle s_{2}\right\rangle$ in terms of the flowing fraction, $f_{2}$, as: 


$$
\left\langle s_{2}\right\rangle=\frac{f_{2}+1-\left(\left(f_{2}+1\right)^{2}-4\left(f_{2}\right)^{2}\right)^{\frac{1}{2}}}{2 f_{2}}
$$

Equations (4) and (5) above are the main results of this paper - the wholly-analytical, correlation-free equation for gas (phase 2) volume fraction in a two-phase flow. Equation (4) is referred to as 'ANSLIP' (meaning analytical slip) in [6]. In terms of dependencies, it is important to note that although the gas volume fraction, $\left\langle s_{2}\right\rangle$, in eqn. (5) is given as a direct function of gas flowing fraction, $f_{2}$, all the other parameters and dependent variables in a multiphase pipe flow such as pipe inclination, diameter, flowing area, densities, viscosities, etc. will be found in the calculation of the gas flowing fraction, $f_{2}$, when eqn. (5) is used in a multiphase flow computational simulator environment (e.g. as in [6]). Therefore, the same variables and parameters involved in the calculation of superficial and mixture velocities of the gas flowing fraction (which are functions of pressure and temperature) contribute implicitly to the calculation of the gas volume fraction.

In terms of mathematical form, although from the derivation above one might initially expect eqn. (4) to be best applicable to high gas volume fraction flows (i.e. churn-annular, annular, annular-mist, wet-gas flows), a plot of $f_{2}$ versus $\left\langle s_{2}\right\rangle$ reveals a broader, behaviour-capturing nature of this equation, as seen in Fig. 1 below. It is notable that the major multiphase flow pattern regions of bubbly (or dispersed) flows at about $\left\langle s_{2}\right\rangle<0.3$, transitional (or slug/churn) flows at about $0.3<\left\langle s_{2}\right\rangle<0.75$, and annular (or separated) flows

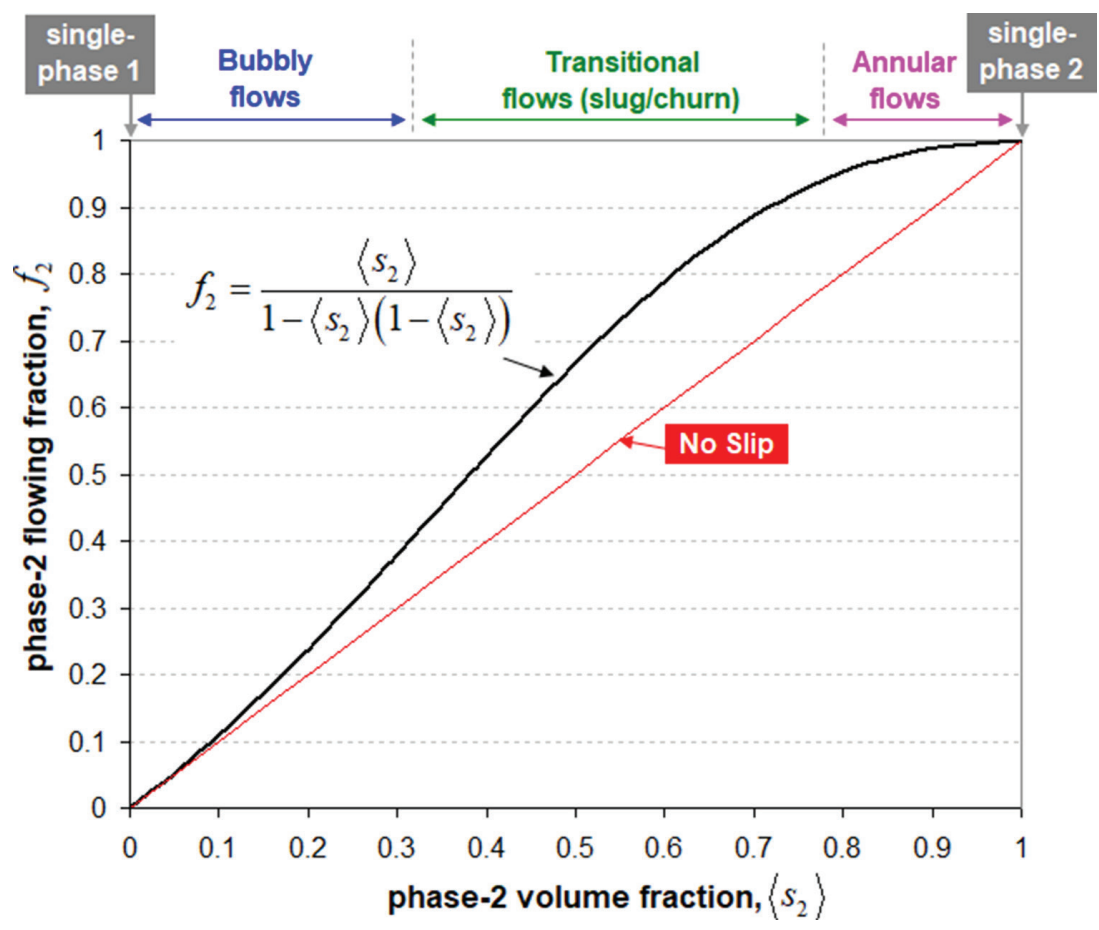

Figure 1: Graphical representation of eqn. (4), the main result of this work. 
at about $\left\langle s_{2}\right\rangle>0.75$ are captured in terms of their relationships to the amount of slip these flow patterns display. The amount of slip in the fractional flow plot of Fig. 1 is depicted by the distance of the model from the no slip line. As can be seen, slug and churn flows will typically have the highest slip followed by annular flows and then bubbly flows. Therefore, by analysing the form of eqn. (4), which is graphically depicted by Fig. 1, we can expect that eqn. (4) will find applicability to a wide range of vertical-up, up-inclined and horizontal multiphase flows, and will have its highest accuracy for high gas volume fraction (or high gas rate) flows.

In terms of the limiting applicability of eqn. (4), we note that the lower gas volume fraction predictions for down-inclined flows are not generally expected to follow eqn. (4) since Fig. 1 shows that the equation does not functional represent the portion of the fractional flow plot below the no slip line at lower gas volume fractions, which indicates regions where phase 1 flows faster than phase 2 . Also, for specific types of gas-liquid flows where the bubbly flows will not exhibit a low-slip (or dispersed bubbly flow) behaviour, such as heavy oil and gas flow or flows with high slip at low gas volume fractions, eqn. (4) is not expected to accurately describe such gas volume fraction behaviours. In this latter case, a gas volume fraction model that allows for high slip at low gas volume fractions should be used (e.g. as found in [8]).

\section{MODEL VALIDATION AGAINST PUBLISHED LAB AND FIELD DATASETS}

For the remainder of this work, we compare the performance of eqn. (4) in both validation tests (below) and in new VFM algorithms for calculating three-phase flow rates (next section).

\subsection{Non-obstructed lab multiphase flows - constant-diameter closed conduit}

In the first validation lab dataset for constant-diameter closed conduits, we select the large database of published experiments given in [9]. In this reference for annular flows, inclusive of churn-annular to annular mist flows, an empirical correlation for gas volume fraction is obtained through the traditional means of non-linear parametric regression. The datasets over which the regressed correlation is drawn spans a gas (phase 2) volume fraction range of $0.7<\left\langle s_{2}\right\rangle<1$, and includes 2,633 datapoints for circular tubes covering macroscale to microscale flow conditions and 40 additional datapoints for non-circular channels.

In order to compare the predictive reliability of eqn. (4) against this database, we plot the empirical correlation of [9] against eqn. (4) for the common experimental flow loop conditions of air density $=1.2 \mathrm{~kg} / \mathrm{m}^{3}$ and water density $=1000 \mathrm{~kg} / \mathrm{m}^{3}$. As is evident from Fig. 2, the very close match of eqn. (4) with this large experimental database (captured by the empirical correlation) demonstrates the accuracy of eqn. (4) and signifies that the time-consuming approach of gathering large amounts of data and regressing upon adjustable parameters of a model can be overcome by simple and mathematically consistent physical arguments (i.e. careful reasoning rather than 'brute force').

\subsection{Obstructed (restricted) lab multiphase flows - variable-diameter chokes \& nozzles}

In the second validation lab dataset for constant-diameter closed conduits, we select the large database of published experiments given in [10]. The datasets of this reference include the full range of flow patterns. In Fig. 3 above, one gas volume fraction and total pressure gradient dataset corresponding to run names of 'WR4.01' to 'WR4.15' in the reference, are selected to show how eqn. (4) is used to first predict the gas volume fraction (Fig. 3a), and then the 


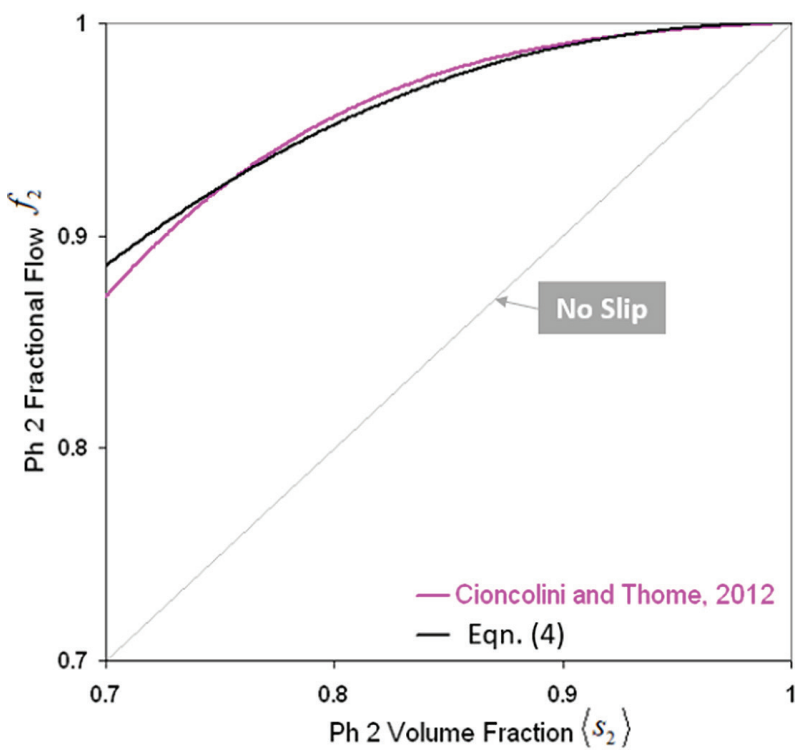

Figure 2: Comparison of the wholly analytical eqn. (4) against a vapor (phase 2) and liquid (phase 1) annular flow empirical correlation [9] inclusive of churnannular to annular-mist boundaries. The underlying experimental database for the correlation contains 2,633 datapoints for circular tubes covering macroscale to microscale flow conditions and 40 additional datapoints for non-circular channels.

(a)

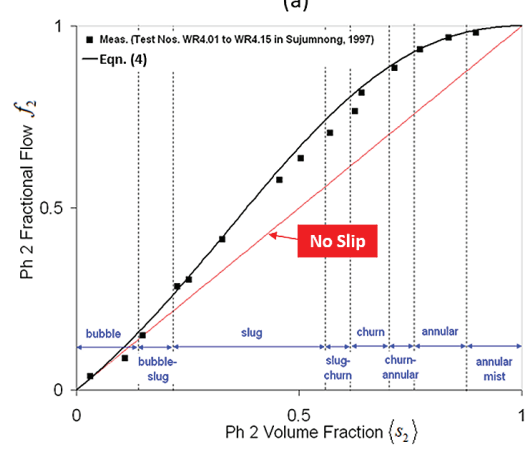

(b)

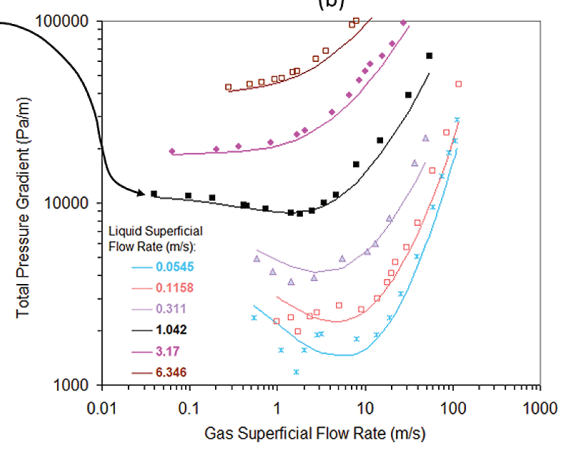

Figure 3: Demonstrating how eqn. (4) can be used to predict both the air volume fraction (in $b$ ) and pressure drops (in $a$ ) of three different sub-critical to critical horizontal airwater choke flow datasets of [11]. Lines are calculations (outlet specified) and points are data.

predicted gas volume fraction is used within the total pressure gradient model for predicting the total pressure gradient dataset at a liquids superficial flow rate of $1.042 \mathrm{~m} / \mathrm{s} \mathrm{(Fig.} \mathrm{3b).} \mathrm{It}$ should be noted how eqn. (4) smoothly transitions through each flow pattern and integrates the different slip velocity transitions as the flow pattern changes. Finally, for comparison, other predictions of pressure gradients using eqn. (4) for the gas volume fraction calculation at different liquid superficial flow rates are additionally shown in Fig. $3 b$. 


\subsection{Obstructed (restricted) lab multiphase flows - variable-diameter choke}

In addition to the constant diameter lab datasets above, we now validate eqn. (4) against three restricted, variable diameter two-phase flow lab datasets sourced from the horizontal multiphase choke flow datasets in [11]. In the reference, we select datasets transitioning the boundary from sub-critical choke flow (runs '21171' and '21170') to critical choke flow (run '21169'). This validation serves an important application of the use of eqn. (4) for gas volume fraction calculations in applications involving critical to sub-critical choke multiphase flows. Note that for the datasets in this section (as well for the remainder of this paper), eqn. (4) is used within the analytical simulator in [6] in which the variable-diameter flow path is carefully discretized into a multi-segmented pipe system that conforms to the flow path. This discretization can be similarly applied to sharp, short obstructions (such as plate orifices or small constrictions) in addition to smooth, long obstructions as given in this choke example and nozzles. Clearly, the character and magnitude of the pressure gradients and phase volume fractions will change in accordance with both the varying segment axial length and varying segment cross-sectional areas along the profile of each differently discretized system.

In Fig. 4 above, each run starts at the specified outlet pressure (varying from low to high pressure) and the gas volume fraction and total pressure gradient calculations are performed

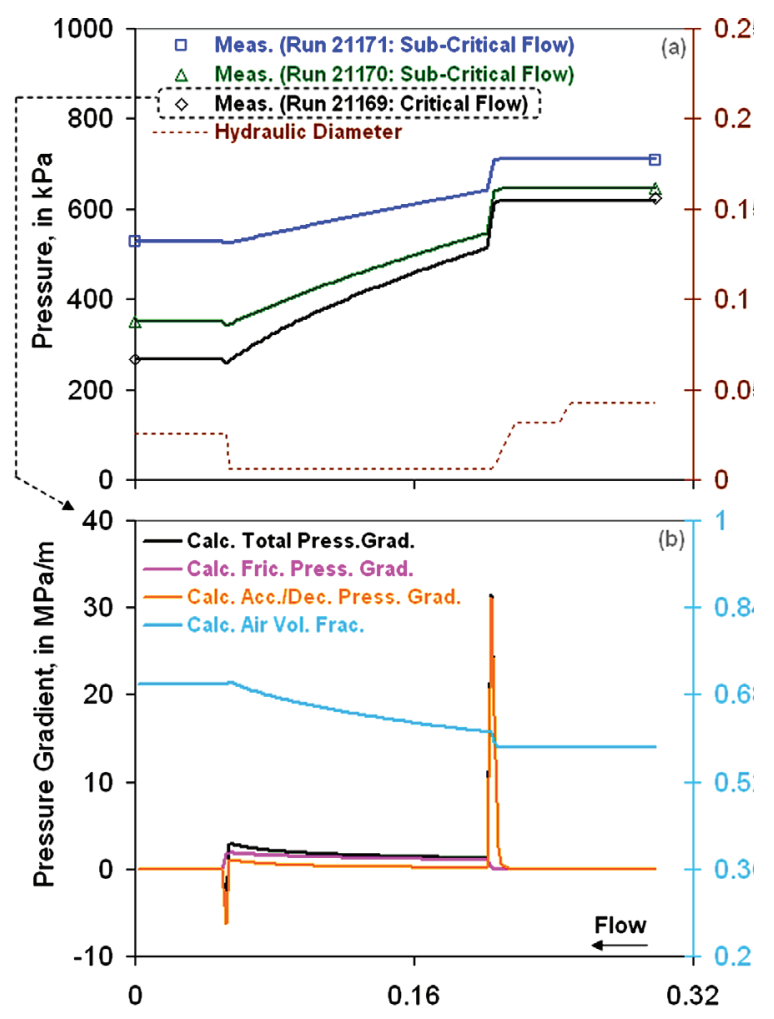

Figure 4: Demonstrating how eqn. (4) can be used to predict both the air volume fraction (in $b$ ) and pressure drops (in $a$ ) of three different sub-critical to critical horizontal airwater choke flow datasets of [11]. Lines are calculations (outlet specified) and points are data. 
along the segmented choke system denoted by a dotted line profile shown by the right axis of Fig. 4a. Note that all subcritical and critical choke multiphase flows are accurately predicted using eqn. (4). It is also important to highlight that in varying diameter flows, as depicted in Fig. 4 above, the convective acceleration/deceleration pressure gradient term will dominate during the contraction and expansion parts of the choke device, where the flow will accelerate (positive pressure gradient) during contractions and decelerate (negative pressure gradient) during expansions. Additionally, the frictional pressure gradient will dominate along the constant small-diameter section of the choke.

\subsection{Validation against published offshore (subsea) flowline field dataset}

Next, in addition to validation comparisons of eqn. (4) against the lab-scale datasets above, we now shift attention to a published, field-scale gas-condensate subsea flowline dataset with real petroleum fluids exhibiting gas dew point mass exchange behaviours (e.g. gas coming out of condensate and condensate dropping out of gas) found in [12]. These tests examine the validity of eqn. (4) in scenarios of changing flowing fractions along the system flow path representing the always-changing superficial gas velocities and mixture velocities during the multiphase flow as pressure and temperature drops.

For this validation, we select the published offshore North Sea Frigg to St. Fergus gascondensate subsea flowline dataset of [12], in which both surface pressure and temperature measurements and pigged flowline condensate volumes measurements are readily available for comparisons against both total pressure gradient and gas volume fraction simulations using eqn. (4). Additionally, this is a very long 226 miles, 32-inch diameter subsea flowline that flows gas and condensate from the Frigg offshore platform to an intermediate 'MCP01' platform and then on to the St, Fergus onshore complex as shown in the elevation (seabed) profile Fig. 5a. The prevailing flow pattern is annular flow with a very high gas-to-condensate ratio (GCR) of $1 \mathrm{MMscf} / \mathrm{BBL}$ at various condensate rates as shown in Fig. 5b. Pressure and temperature data are available on surface at the Frigg platform and the St. Fergus complex and sometimes available at the intermediate platform for each for the condensate flow rates shown in Fig. 5 b.

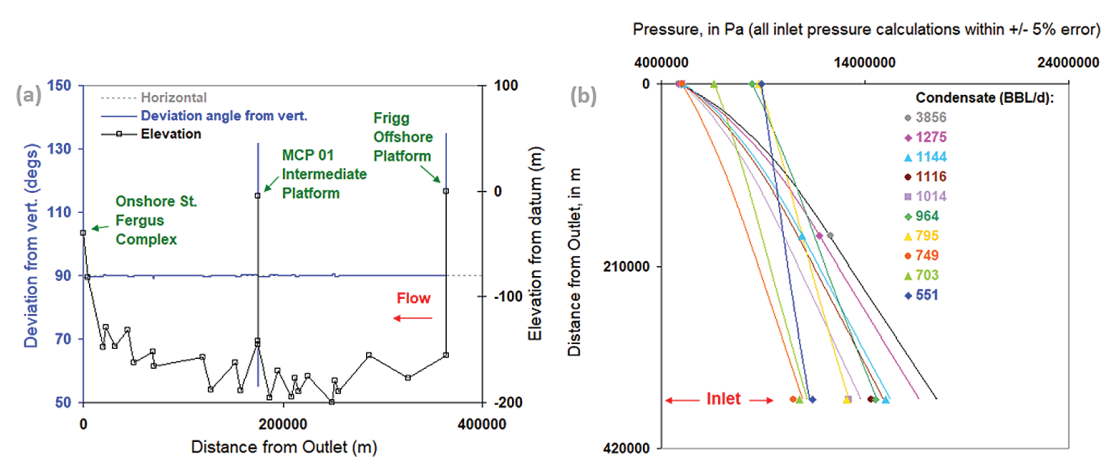

Figure 5: Demonstrating how eqn. (4) can be used to predict both the condensate holdup (or condensate volume fraction) and total pressure drops in $b$, for the elevation profile of the 226 miles long, 32-inch diameter North Sea subsea Frigg flowline [12] in (a). In $(b)$, lines are our analytical simulations and points are pressure gauge data. 
As is clear from the results of Fig. 5b, eqn. (4) is ideally suited for this annular flow dataset and yields a high accuracy for all the reported pressure gauge data within $+/-5 \%$ error. For these data, all simulations started with the fixed outlet pressure and the pressure profiles and inlet flowline pressures were predicted using eqn. (4).

\section{NEW ALGORITHM FOR CALCULATING FLOWLINE FLOW RATES USING KNOWN SURFACE PRESSURE AND TEMPERATURE DIFFERENCES}

In this final validation section, we will utilize eqn. (4) in a new algorithm for field-scale virtual multiphase flow metering calculations presented below by utilizing the pressure and temperature differences along a subsea pipeline to calculate its producing condensate and gas flow rates. For this calculation, we select one of the flow rates of the North Sea Frigg subsea flowline dataset from the previous section, i.e. the dataset at condensate rate of $1144 \mathrm{BBL} / \mathrm{d}$. The corresponding outlet MCP01 platform pressure of $10.92 \mathrm{MPa}$ and temperature of $5.5^{\circ} \mathrm{C}$ will be used as the starting point in our simulations. For the given inlet Frigg platform pressure of $15 \mathrm{MPa}$ and temperature of $47^{\circ} \mathrm{C}$, our goal then is to find the flow rates that yield the minimum absolute difference between the calculated pressure drop between the Frigg and MCP01 platforms $\left(\Delta \mathrm{P}_{\text {calc }}\right)$ using eqn. (4) and the measured pressure drop between these platforms of $15 \mathrm{MPa}-$ $10.92 \mathrm{MPa}=4.08 \mathrm{MPa}\left(\Delta \mathrm{P}_{\text {meas }}\right)$. This absolute difference is shown on the y-axis of Fig. 6 below.

In setting up this dataset for VFM simulations, we note the total pipeline measured distance (MD) from the Frigg platform to the MCP01 platform $=188,400 \mathrm{~m}$, a horizontal pipeline profile assumption is used, a linear flowing temperature gradient assumption is used, condensate gravity $=70^{\circ} \mathrm{API}$, gas gravity $=0.68$ (Air $=1.0$ ) and the internal pipeline diameter $=0.7747 \mathrm{~m}$. For our VFM algorithm, we first specify increasing condensate rates in 50-BBL/d increments from 100 to $2000 \mathrm{BBL} / \mathrm{d}$ at GCR $=0.5 \mathrm{MMscf} / \mathrm{BBL}$ (simulation run \# 1 to 39 of Fig. 6), then at the same condensate increments at GCR $=1 \mathrm{MMscf} / \mathrm{BBL}$ (simulation run \# 40 to 78 of Fig. 6), and finally at the same condensate increments at GCR $=1.5 \mathrm{MMscf} /$ BBL (simulation run \# 79 to 117 of Fig. 6).

As clearly seen in Fig. 6, the unknown flow rates can be uniquely found for the presented dataset. Simulation number 61 represents the condition of $1150 \mathrm{BBL} / \mathrm{d}$ and GCR of $1 \mathrm{MMscf} /$

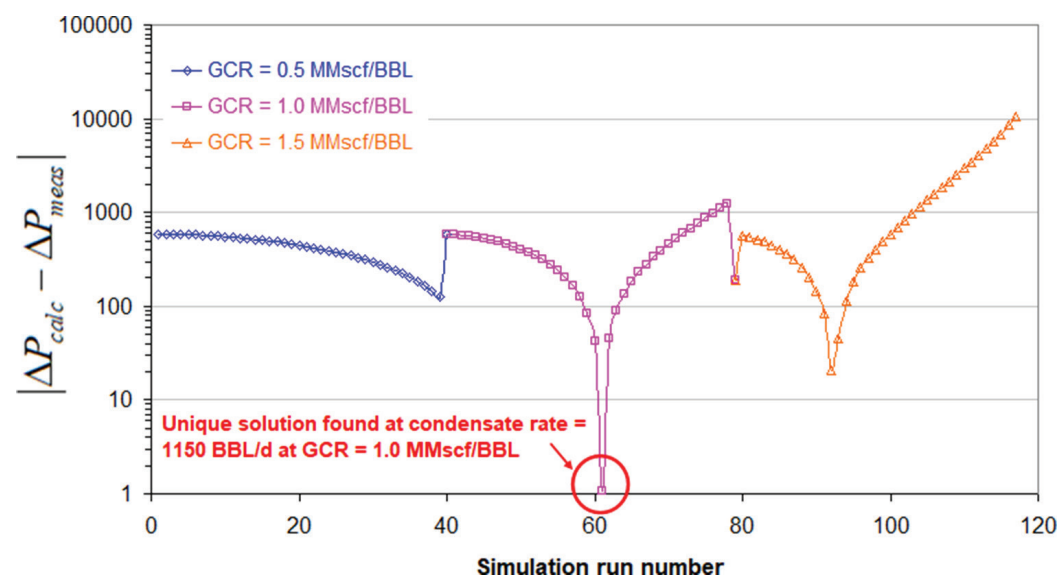

Figure 6: Demonstrating how eqn. (4) can be used to predict the condensate and gas flow rates of one published dataset of the North Sea Frigg subsea flowline in [12]. 
BBL, which is very close to the actual rates. This procedure of specifying the flow rate combinations and determining the minimum value of $\left|\Delta P_{\text {calc }}-\Delta P_{\text {meas }}\right|$ can be applied to any flowline.

Last, it should be noted that a similar VFM algorithm to that described above can be applied to oilfield wellbores for predicting three-phase flow rates at the wellhead. In such applications, the calculations of flowing bottom hole pressure (FBHP) from the outlet specified conditions at the wellhead using eqn. (4), i.e. $P_{w f, c a l c}$, will be compared to the FBHP specified from an inflow performance relationship (IPR) that relates the FBHP to the gas-tooil ratio (GOR), water cut, total fluids productivity index (PI) and an averaged reservoir pressure, i.e. $P_{w f, I P R}$. The absolute difference to be minimized in this scenario will be $\left|P_{w f, c a l c}-P_{w f, I P R}\right|$. Additionally, in contrast to the flowline VFM application above, in the wellbore VFM application, only the gas rate needs to be specified in increments from a chosen low value to high value since the corresponding oil rates will be provided from the given GOR and the corresponding water rates will be provided from the given water cut.

\section{CONCLUSIONS}

The validation results of the lab and field case studies given in this work clearly show the predictive value of using the presented correlation-free, wholly-analytical gas volume fraction model (eqn. 4) that is smooth, continuous and differentiable, in that unique phase flow rate combinations result when used in conjunction with analytical multiphase flow modelling methods. This is a practically significant finding that is useful for both forward models (using phase flow rates to calculate pressure gradient) as well as inverse models (VFM optimization algorithms using pressure gradient to calculate phase flow rates). The presented model will find ideal use in virtual multiphase flow meter and production allocation field applications in the petroleum industry for its differentiated ability to achieve improved reliability of flow rate predictions.

\section{REFERENCES}

[1] Corneliussen, S., Couput, J.P., Dahl, E., Dykesteen, E., Frøysa, K. E., Malde, E. ... \& Tunheim, H., Handbook of multiphase flow metering, Norwegian Society for Oil and Gas Measurement (NFOGM), Revision, 2, March 2005.

[2] Falcone, G., Hewitt, G.F., Alimonti, C. \& Harrison, B., Multiphase flow metering: current trends and future developments. Journal of Petroleum Technology, 54(4), 2002. https://doi.org/10.2118/74689-ms

[3] Mwalyepelo, J.Y., Analysis of Selected Multiphase Metering Concepts, M.S. Thesis, Norwegian University of Science and Technology, July 2015.

[4] Whitfield, S., Production monitoring gets smarter with virtual flow metering. Oil and Gas Facilities, May 2019.

[5] Nagoo, A.S., Analytical Modeling of Multidirectional Entrainment in Three-Phase Gas-Liquid-Liquid Flows with Oilfield Applications in High Gas Rate Wellbores and Flowlines, 10th Intl. Conf. Multiphase Flow, Rio De Janeiro, Brazil, 2019.

[6] Nagoo, A.S., Multiphase Analytical Prediction Engine (MAPe) - an analytical multiphase flow simulator, http://www.nagoo-associates.com, 2018.

[7] Nagoo, A.S. \& Sharma, M.M., Finer theoretical aspects of the drift flux family of equations for simulating averaged volume fraction in multiphase flows. International Journal of Computational Methods and Experimental Measurements, 6(2), pp. 240-250, 2017. https://doi.org/10.2495/cmem-v6-n2-240-250 
[8] Woldesmayat, M.A. \& Ghajar, A.J., Comparison of void fraction correlations for different flow patterns in horizontal and upward inclined pipes. International Journal of Multiphase Flow, 33(4), pp. 347-370, 2007. https://doi.org/10.1016/j.ijmultiphaseflow.2006.09.004

[9] Cioncolini, A. \& Thome, J.R., Void fraction prediction in annular two-phase flow. International Journal of Multiphase Flow, 43, pp. 72-84, 2012. https://doi.org/10.1016/j. ijmultiphaseflow.2012.03.003

[10] Sujumnong, M., Heat Transfer, Pressure Drop and Void Fraction in Two-Phase, TwoComponent Flow in a Vertical Tube, Ph.D. Dissertation, University of Manitoba, 1997.

[11] Pilehvari, A.A., Experimental Study of Subcritical Two-Phase Flow Through Wellhead Chokes, Research Report, Tulsa U. Fluid Flow Projects, September 1980.

[12] Lagiere, M., Miniscloux, C. \& Roux, A., Computer two-phase flow model predicts pipeline pressure and temperature profiles, Oil and Gas Facilities, April 1984. 